\title{
Fully Digital 1-D, 2-D and 3-D Multiscroll Chaos as Hardware Pseudo Random Number Generators
}

\author{
Abhinav S. Mansingka \\ Electrical Engineering Program, \\ King Abdullah University of \\ Science and Technology, \\ Thuwal, Saudi Arabia 23955-6900. \\ Email: abhinav.mansingka@kaust.edu.sa
}

\author{
Ahmed G. Radwan \\ Department of Engineering Mathematics, \\ Faculty of Engineering, \\ Cairo University, \\ Giza, Egypt. \\ Email: agradwan@ieee.org
}

\author{
Khaled N. Salama \\ Electrical Engineering Program, \\ King Abdullah University of \\ Science and Technology, \\ Thuwal, Saudi Arabia 23955-6900. \\ Email: khaled.salama@kaust.edu.sa
}

\begin{abstract}
This paper introduces the first fully digital implementation of 1-D, 2-D and 3-D multiscroll chaos using the sawtooth nonlinearity in a 3rd order ODE with the Euler approximation. Systems indicate chaotic behaviour through phase space boundedness and positive Lyapunov exponent. Low-significance bits form a PRNG and pass all tests in the NIST SP. 800-22 suite without post-processing. Real-time control of the number of scrolls allows distinct output streams with 2-D and 3-D multiscroll chaos enabling greater controllability. The proposed PRNGs are experimentally verified on a Xilinx Virtex 4 FPGA with logic utilization less than $1.25 \%$, throughput up to $5.25 \mathrm{Gbits} / \mathrm{s}$ and up to $\mathbf{5 1 2}$ distinct output streams with low cross-correlation.
\end{abstract}

Keywords-Chaos; nonlinear systems; digital circuits; pseudo random number generator; field programmable gate array;

\section{INTRODUCTION}

Chaotic oscillators have been emphasized for applications in random number generation [1]-[6] wherein the repeatable, reliable and high-performance chaotic sources required for these applications [7] are difficult to realize using analog circuits with chaos degradation due to established issues of PVT-sensitivity [8]. In particular, multi-scroll chaos generators have been exhaustively studied [9]-[12], wherein the limitations on the dynamic range of analog components and low supply voltages has restricted the number of scrolls that can be realized due to complex circuitry needed for control [13] unless mixed-signal approaches are used [14]. Register transfer level design has been shown to enable reliable and fully digital chaotic oscillators [15]-[17] for direct application in digital systems. Analog limitations are eliminated and repeatability is enabled through ease of register initialization. However, previous fully digital implementations of the chaotic logistic map had very high hardware requirements [2], [6] and diminished throughput. Jerk-equation based chaotic systems [18] have been digitally implemented and assessed for the effect of bus width and delay elements on the chaotic response [15] and optimized for random number generation [5].

Using a fully digital pipelined architecture, this paper implements for the first time, 1-D, 2-D and 3-D multiscroll chaos using sawtooth nonlinearities with controllable bounds. Paritcularly, this is the first digital implementation of 2-D and 3 -D grid-scroll chaos. The Euler approximation is used to
TABLE I

1-D, 2-D AND 3-D Multiscroll ChaOs: System ODEs AND EQUILIBRIUM POINTS.

\begin{tabular}{c|cc}
\hline & System ODE & Equlibrium Points \\
\hline \multirow{2}{*}{ 1D } & $\dot{X}=Y$ & $(i+0.5,0,0)$ \\
& $\dot{Y}=Z$ & $i \in\left[L_{x}, U_{x}\right)$ \\
\hline \multirow{2}{*}{ 2D } & $\dot{Z}=-\alpha[F(X)+Y+Z]$ & $(i+0.5, j+0.5,0)$ \\
& $\dot{X}=F(Y)$ & $i \in\left[L_{x}, U_{x}\right)$ \\
& $\dot{Y}=Z$ & $j \in\left[L_{y}, U_{y}\right)$ \\
\hline \multirow{2}{*}{ 3D } & $\dot{Z}=-\alpha[F(X)+F(Y)+Z]$ & $(i+0.5, j+0.5, k+0.5)$ \\
& $\dot{X}=F(Y)$ & $i \in\left[L_{x}, U_{x}\right)$ \\
& $\dot{Y}=F(Z)$ & $j \in\left[L_{y}, U_{y}\right)$ \\
& $\dot{Z}=-\alpha[F(X)+F(Y)+F(Z)]$ & $k \in\left[L_{z}, U_{z}\right)$ \\
\hline
\end{tabular}

numerically solve the system of 3rd order ordinary differential equations (ODEs). These are then implemented as pseudo random number generators (PRNGs) without any additional post-processing by truncating high-significance bits. This eliminates short-term predictability [5] and here also suppresses cross-correlation and enables passage of all NIST SP. 80022 randomness tests. Extension of scrolls to higher dimensions enables enhanced programmability and higher nonlinear complexity. The PRNGs are experimentally verified on a Xilinx Virtex 4 FPGA with logic utilizations not exceeding $1.25 \%$, throughput verified up to $5.25 \mathrm{Gbits} / \mathrm{s}$, and 3 times greater area-efficiency than previous work while providing high nonlinear complexity through multiscroll chaos and up to 512 controllable output streams with low cross-correlation.

\section{Digital Implementation}

Multiscroll chaos can be described using a 3rd order ODE using a sawtooth nonlinearity $F(X)$ with integer lower bound $L$, upper bound $U$ and floor function $\lfloor X\rfloor$ given by:

$$
F(X)= \begin{cases}X-U-0.5 & X \in(U+0.5, \infty) \\ X-L-0.5 & X \in(-\infty, L+0.5) \\ X-\lfloor X\rfloor-0.5 & X \in[L+0.5, U+0.5]\end{cases}
$$


Essentially, multidimensional scrolls arise from expanding the nonlinearity in $\mathrm{X}$ [14] to $\mathrm{Y}$ and $\mathrm{Z}$. Table I specifies the ODEs for 1-D, 2-D and 3-D multiscroll chaos. At each equilibrium point $(\dot{X}, \dot{Y}, \dot{Z})=(0,0,0)$, all three systems have the same Jacobian and characteristic equations:

$$
\begin{gathered}
J=\left[\begin{array}{ccc}
0 & 1 & 0 \\
0 & 0 & 1 \\
-\alpha & -\alpha & -\alpha
\end{array}\right] \\
s^{3}+\alpha s^{2}+\alpha s+\alpha=0
\end{gathered}
$$

giving eigenvalues of $(-0.9687,0.0156 \pm 0.9836 i)$ for $\alpha=$ 0.9375 , with one negative real eigenvalue and a pair of complex conjugate eigenvalues with positive real parts, a saddle point of index 2 that is necessary for the formation of scrolls at each equilibrium point, verified through numerical simulation. The flow is dissipative as the sum of eigenvalues is negative. The Euler approximation (with step size $h$ ) gives the numerical solution according to Fig. 1(a) for 3-D multiscroll chaos:

$$
\begin{aligned}
X_{t+h} & =X_{t}+h\left[\dot{X}_{t}\right] \\
Y_{t+h} & =Y_{t}+h\left[\dot{Y}_{t}\right] \\
Z_{t+h} & =Z_{t}+h\left[\dot{Z}_{t}\right]
\end{aligned}
$$

Registers $\{\mathrm{X}, \mathrm{Y}, \mathrm{Z}\}$ store the system state while combinational logic determines the next state. A 32-bit fixed-point two's complement representation is used with 5 bits for the sign and integer part and 27 bits for the fraction part. The stepsize is $h=2^{-3}$, simplifying to a hardcoded right-shift. The system parameter $(\alpha=0.9375)$ is realized by noting that $0.9375=1-2^{-4}$. For 1-D and 2-D multiscroll chaos, $F(Z)$ and $F(Y)$ modules are eliminated as required by the ODEs and replaced by wires. While many parameters can be made controllable in a digital multiscroll system, varying only the number of scrolls incurs the smallest performance penalty [16]. The hardware implementation of the sawtooth function in (1) is shown in Fig. 1(b) with the resulting output in Fig. 1(c). $L$ and $U$ are integer-valued, with $L=-6$ fixed and a controllable 3-bit $U(U \in[-4,3])$, two less than the integer width to prevent arithmetic overflow. Sign-extension by 2 bits matches $U$ with the integer width and one-padding to the right by 1 bit adds 0.5 . This is fully scalable and more control can be added simply by using a wider integer width (thus wider $L$ and $U$ ) to accommodate more scrolls. The number of scrolls in each dimension $d \in\{x, y, z\}$ is given as:

$$
N_{\mathrm{d}}=U_{\mathrm{d}}-L_{\mathrm{d}}+1=U_{\mathrm{d}}+7, \quad U_{\mathrm{d}} \in[-4,3]
$$

ranging between 3 and 10 and dependent only on the upper bound of the sawtooth nonlinearity in that dimension.

\section{Chaotic Response}

Attractors (X-Y or Y-Z phase plots) from the digitally implemented 1-D, 2-D and 3-D multiscroll systems are shown in Fig. 2 with all of them showing excellent phase-space boundedness. It is important to note that since this paper introduces finite precision numerical solutions of what are infiniteprecision continuous-time ODEs, the trajectories produced by the digital implementations are, by definition, pseudochaotic and approximate the chaotic behavior expected from

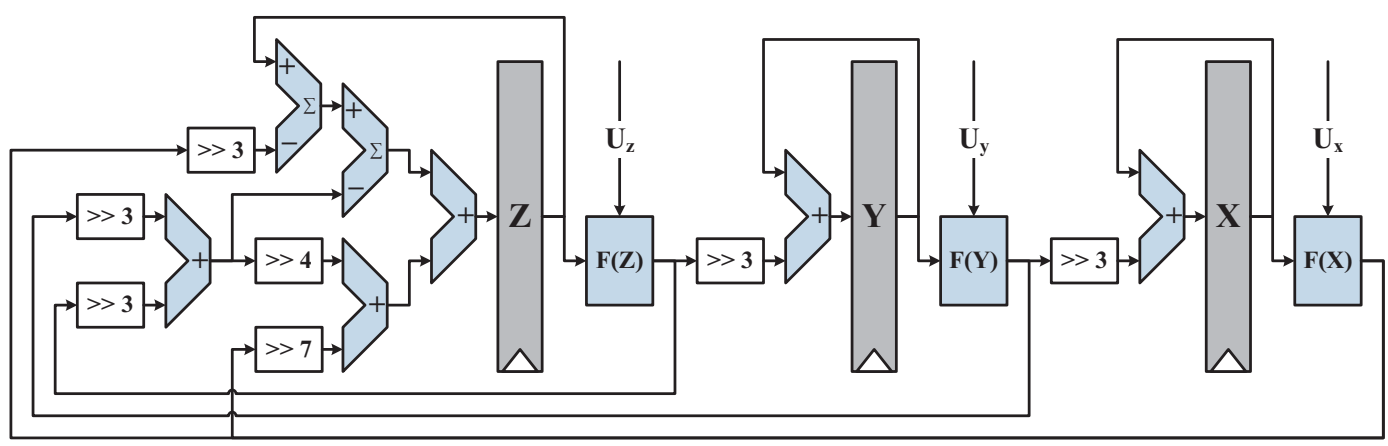

(a)

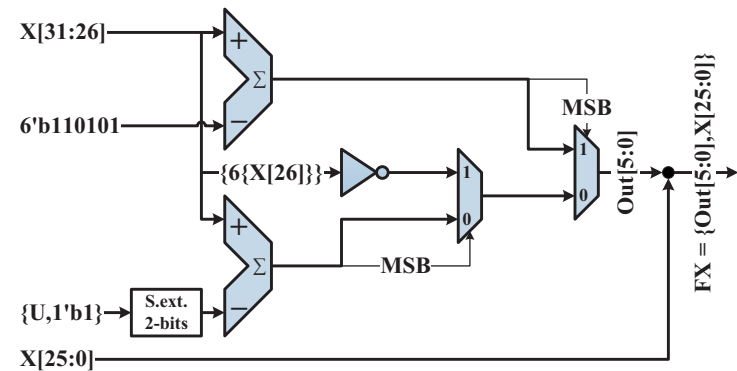

(b)

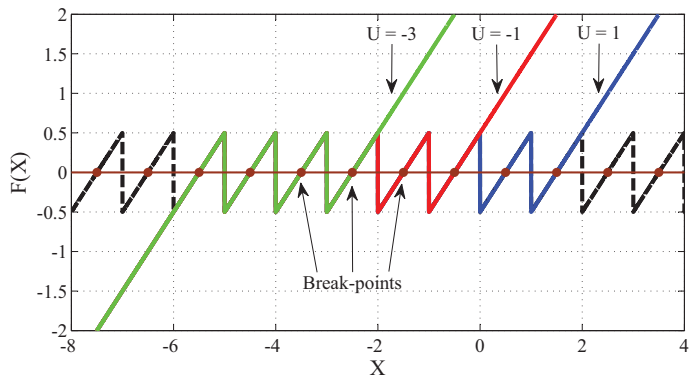

(c)

Fig. 1. (a) Schematic for 3-D multiscroll chaos: 2-D and 1-D multiscrolls can be obtained by replacing $F(Z)$ and $F(Y)$ with wires based on the ODEs, (b) Digital realization of the sawtooth function and (c) the bounded sawtooth function from (1) with $L=-6$ and $U=\{-3,-1,1\}$. 


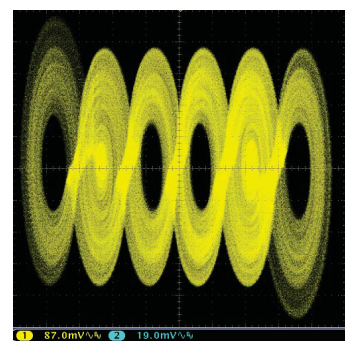

(a)

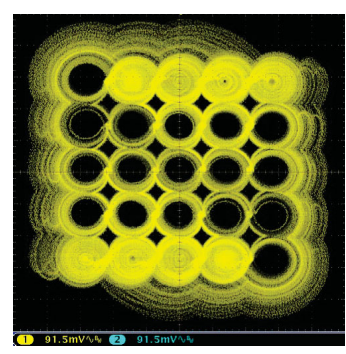

(c)

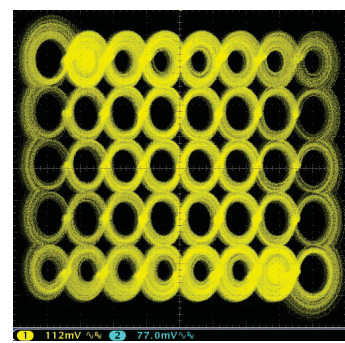

(b)

(d)

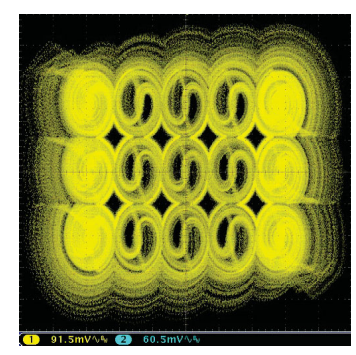

Fig. 2. Oscilloscope trace of experimentally obtained (a) X-Y attractor with $U_{x}=-1\left[1-\mathrm{D}\right.$ : 6 scrolls], (b) X-Y attractor with $\left(U_{x}, U_{y}\right)=$ $(1,-2)[2-\mathrm{D}: 8 \times 5$ scrolls $]$ and (c)-(d) X-Y and Y-Z attractors with $\left(U_{x}, U_{y}, U_{z}\right)=(-2,-2,-4)[3-\mathrm{D}: 5 \times 5 \times 3$ scrolls $]$.

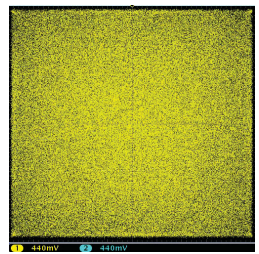

(a)

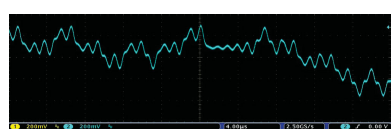

(d)

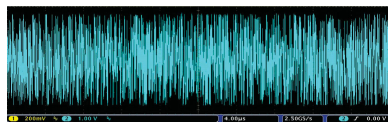

(f)

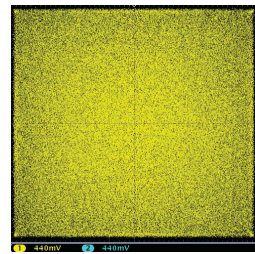

(b)

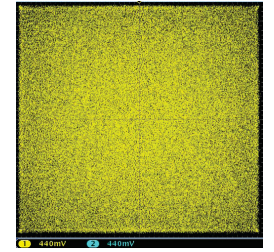

(c)

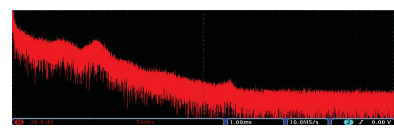

(e)

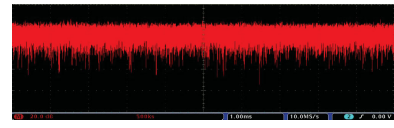

(g)

Fig. 3. Oscilloscope trace of 1-D multiscroll (a)-(c) X-Y, Y-Z and X-Z attractors after truncation of defective bits, time series and FFT of $X$ for (c)-(d) original output and (e)-(f) after truncation of defective bits.

analog systems. However, the pseudo-chaos can be verified by calculating the maximum Lyapunov exponent (MLE), wherein a positive MLE verifies the existence of chaotic dynamics. Mathematically, this value expresses the effect of an arbitrarily small change in initial conditions on the overall divergence in the output solution over time, according to $\delta S(t) \approx e^{\lambda t} S(\delta t)$. Using software based on [19], the MLEs are 0.138, 0.230, and 0.259 for 1 -D $(6$ scrolls $), 2-D(8 \times 5$ scrolls $)$ and $3-D$ $(5 \times 5 \times 3$ scrolls $)$ multiscroll systems and thus indicate chaotic dynamics. The MLE generally increases for a larger number of scrolls [16] and remains positive.

TABLE II

NIST SP. 800-22 Test Results AND AREA/Performance Results on A XILINX VIRTEX 4 FPGA

\begin{tabular}{|c|c|c|c|c|c|c|}
\hline \multicolumn{7}{|c|}{ NIST SP. 800-22 Results } \\
\hline & \multicolumn{2}{|c|}{ 1-D } & \multicolumn{2}{|c|}{$2-\mathrm{D}$} & \multicolumn{2}{|c|}{$3-\mathrm{D}$} \\
\hline & PV & PP & PV & PP & PV & PP \\
\hline Monobits & $\checkmark$ & 0.96 & $\checkmark$ & 1.00 & $\checkmark$ & 0.98 \\
\hline Block Frequency & $\checkmark$ & 0.93 & $\checkmark$ & 0.98 & $\checkmark$ & 1.00 \\
\hline Cumulative Sums & $\checkmark$ & 0.98 & $\checkmark$ & 1.00 & $\checkmark$ & 0.98 \\
\hline Runs & $\checkmark$ & 1.00 & $\checkmark$ & 1.00 & $\checkmark$ & 1.00 \\
\hline Longest Run & $\checkmark$ & 0.98 & $\checkmark$ & 1.00 & $\checkmark$ & 1.00 \\
\hline Rank & $\checkmark$ & 1.00 & $\checkmark$ & 1.00 & $\checkmark$ & 0.98 \\
\hline FFT & $\checkmark$ & 0.98 & $\checkmark$ & 1.00 & $\checkmark$ & 0.98 \\
\hline N. O. Template & $\checkmark$ & 0.99 & $\checkmark$ & 0.99 & $\checkmark$ & 0.99 \\
\hline O. Template & $\checkmark$ & 0.98 & $\checkmark$ & 0.98 & $\checkmark$ & 1.00 \\
\hline Universal & $\checkmark$ & 1.00 & $\checkmark$ & 0.96 & $\checkmark$ & 0.96 \\
\hline Approx. Entropy & $\checkmark$ & 1.00 & $\checkmark$ & 0.96 & $\checkmark$ & 1.00 \\
\hline Random Excursion & $\checkmark$ & 1.00 & $\checkmark$ & 0.99 & $\checkmark$ & 0.98 \\
\hline Random Excursion V. & $\checkmark$ & 1.00 & $\checkmark$ & 0.98 & $\checkmark$ & 0.99 \\
\hline Serial & $\checkmark$ & 0.98 & $\checkmark$ & 0.99 & $\checkmark$ & 0.99 \\
\hline Linear Complexity & $\checkmark$ & 0.98 & $\checkmark$ & 1.00 & $\checkmark$ & 1.00 \\
\hline Overall Result & & & & & & ss \\
\hline \multicolumn{7}{|c|}{ Experimental Results on the Xilinx Virtex 4 FPGA } \\
\hline Total LUTs & \multicolumn{2}{|c|}{251} & \multicolumn{2}{|c|}{271} & \multicolumn{2}{|c|}{295} \\
\hline Total FFs & \multicolumn{2}{|c|}{96} & \multicolumn{2}{|c|}{96} & \multicolumn{2}{|c|}{96} \\
\hline Gate Count (Gc) & \multicolumn{2}{|c|}{2776} & \multicolumn{2}{|c|}{2936} & \multicolumn{2}{|c|}{3128} \\
\hline PRNG Bits $\left(N_{P R N G}\right)$ & \multicolumn{2}{|c|}{45} & \multicolumn{2}{|c|}{45} & \multicolumn{2}{|c|}{45} \\
\hline Frequency $[\mathrm{MHz}]$ & \multicolumn{2}{|c|}{116.73} & \multicolumn{2}{|c|}{113.69} & \multicolumn{2}{|c|}{111.29} \\
\hline Throughput $[\mathrm{Mb} / \mathrm{s}]$ & \multicolumn{2}{|c|}{5253} & \multicolumn{2}{|c|}{5116} & \multicolumn{2}{|c|}{5008} \\
\hline FOM [Gc/Th.] & \multicolumn{2}{|c|}{1.89} & \multicolumn{2}{|c|}{1.74} & \multicolumn{2}{|c|}{1.60} \\
\hline
\end{tabular}

TABLE III

STATISTICS OF THE CROSS-CORRELATIONS BETWEEN OUTPUT STREAMS FOR DIFFERENT CONTROL PARAMETERS FOR 1-D, 2-D AND 3-D MULTISCROLL SYSTEMS.

\begin{tabular}{c|c|ccccc}
\hline & & Max. & Min. & Mean & Median & Std. Dev. \\
\hline \hline \multirow{2}{*}{ 1-D } & $\mathrm{X}$ & 0.1742 & $9.85 e-04$ & 0.0347 & 0.0285 & 0.03642 \\
& $\mathrm{Y}$ & 0.0226 & $2.58 e-06$ & 0.0074 & 0.0077 & 0.00541 \\
& $\mathrm{Z}$ & 0.0240 & $1.51 e-04$ & 0.0085 & 0.0067 & 0.00669 \\
& $\mathrm{R}$ & 0.0035 & $8.75 e-05$ & 0.0011 & 0.0008 & 0.00099 \\
\hline \multirow{2}{*}{ 2-D } & $\mathrm{X}$ & 0.1120 & $1.01 e-05$ & 0.0198 & 0.0152 & 0.01753 \\
& $\mathrm{Y}$ & 0.1173 & $4.67 e-06$ & 0.0207 & 0.0172 & 0.01625 \\
& $\mathrm{Z}$ & 0.0278 & $5.61 e-06$ & 0.0055 & 0.0047 & 0.00417 \\
& $\mathrm{R}$ & 0.0051 & $9.04 e-07$ & 0.0013 & 0.0011 & 0.00100 \\
\hline \multirow{3}{*}{$3-\mathrm{D}$} & $\mathrm{X}$ & 0.1442 & $8.30 e-09$ & 0.0090 & 0.0059 & 0.01025 \\
& $\mathrm{Y}$ & 0.1467 & $6.32 e-08$ & 0.0097 & 0.0061 & 0.01120 \\
& $\mathrm{Z}$ & 0.1665 & $4.94 e-08$ & 0.0110 & 0.0067 & 0.01305 \\
& $\mathrm{R}$ & 0.0098 & $5.32 e-10$ & 0.0013 & 0.0011 & 0.00095 \\
\hline
\end{tabular}

\section{IMPLEMENTATION AS PRNGS}

The positive MLE of the digitally implemented multiscroll systems indicates that the output is unpredictable over the long term and thus is suitable to be implemented as a pseudo random number generator. Discarding the high-significance bits greatly suppresses short-term predictability [5] and enables the passage of all tests in the NIST SP. 800-22 statistical test suite [20]. In the 1-D, 2-D and 3-D case, the high 17 bits from each of the outputs $\mathrm{X}, \mathrm{Y}$ and $\mathrm{Z}$ are discarded. The low 15 bits from $\mathrm{X}, \mathrm{Y}$ and $\mathrm{Z}$ are concatenated and form a PRNG for which the NIST SP. 800-22 test results, area utilization 
TABLE IV

COMPARISON WITH PREVIOUSLY REPORTED CHAOS-BASED PRNGS.

\begin{tabular}{|c|c|c|c|c|c|}
\hline \multicolumn{2}{|c|}{ System } & \multirow{2}{*}{$\begin{array}{c}\begin{array}{c}\text { Area } \\
(\mathbf{G c})\end{array} \\
3988\end{array}$} & \multirow{2}{*}{$\begin{array}{c}\begin{array}{l}\text { T.put } \\
(\mathbf{M b} / \mathbf{s})\end{array} \\
200\end{array}$} & \multirow{2}{*}{$\begin{array}{c}\text { FOM } \\
0.05\end{array}$} & \multirow{2}{*}{$\begin{array}{c}\text { NIST } \\
\text { Pass }\end{array}$} \\
\hline Addabbo, 2007 [1] & Rényi Map & & & & \\
\hline Chen, 2010 [2] & Log. Map & 9622 & 200 & 0.02 & Pass \\
\hline $\mathrm{Li}, 2010[3]$ & Log. Map & 9136 & 200 & 0.02 & Fail \\
\hline Chen, 2010 [4] & Log. Map & 31655 & 3200 & 0.10 & Pass \\
\hline Zidan, 2011 [5] & ODE & 2464 & 1180 & 0.48 & Pass \\
\hline $\mathrm{Li}, 2012[6]$ & Log. Map & 11903 & 6400 & 0.54 & Pass \\
\hline \multicolumn{2}{|c|}{ This Work } & & & & \\
\hline 1-D Multiscroll & ODE & 2776 & 5253 & 1.89 & Pass \\
\hline 2-D Multiscroll & ODE & 2936 & 5116 & 1.74 & Pass \\
\hline 3-D Multiscroll & ODE & 3128 & 5008 & 1.60 & Pass \\
\hline
\end{tabular}

and throughput on a Xilinx Virtex 4 XC4VSX35-10FF668 FPGA (30,720 LUTs, 30,720 FFs) are shown in Table II. All three systems exhibit excellent statistical properties with experimentally verified throughput up to $5.25 \mathrm{Gbits} / \mathrm{s}$ and LUT utilization not exceeding $1.25 \%$ and FF utilization not exceeding $0.45 \%$ of total resources available on the FPGA. A gate count is estimated as $G c=8 \times(L U T s+F F s)$ and the area efficiency is assessed through a figure of merit determined as $F O M=\left(N_{P R N G} \times f_{C L K}\right) / G c$. As expected, there is an overhead in area when extending to 2-D and 3-D multiscroll chaos. However, this extension to multi-dimensions gives a multifold increase in the number of distinct output streams from 8 to 64 and 512 respectively on the basis of the 3-bit control parameter assigned to the upper bound in each dimensions. Oscilloscope traces of the X-Y, Y-Z and X$\mathrm{Z}$ attractors after truncation and time series and frequency response of the $\mathrm{X}$-variable before and after truncation are shown in Fig. 3. Clearly, the discarding defective bits gives a far more random output and a uniform spectrum. Table III summarizes the statistics of the magnitude of cross-correlation coefficients from each system for different control parameters (8 streams for 1-D, 64 streams for 2-D and 512 streams for 3-D). The cross-correlations of the native $\mathrm{X}, \mathrm{Y}$ and $\mathrm{Z}$ outputs of each system show very high correlation that is completely suppressed in the corresponding PRNG output (R). The average cross-correlation is on the order of $10^{-3}$. The three multiscroll chaos-based PRNGs implemented in this paper are compared to previous work in Table IV. Clearly, the implemented systems have lower area than all the implementations of 1-D chaotic maps. On that basis, the proposed systems outperform previous literature from an areaefficiency perspective given the $\sim 5$ Gbits/s throughput and are approximately 3 times more area efficient than the best previous implementation.

\section{CONCLUSION}

Fully digital implementations of 1-D, 2-D and 3-D multiscroll chaos through the sawtooth nonlinearity are introduced using the Euler approximation. The output indicates chaotic behaviour through positive maximum Lyapunov exponent and phase-space boundedness. Furthermore, the number of scrolls in all three dimensions can be manipulated in real-time through a controllable upper bound. The low-significant bits from each dimension are concatenated to yield PRNGs that pass the NIST SP. 800-22 testing suite with throughput up to $5.25 \mathrm{Gbits} / \mathrm{s}$ and can produce up to 512 distinct streams with low crosscorrelation. These have useful applications in chaos-based digital communication and encryption systems.

\section{REFERENCES}

[1] T. Addabbo, M. Alioto, A. Fort, A. Pasini, S. Rocchi, and V. Vignoli, "A class of maximum-period nonlinear congruential generators derived from the Rènyi chaotic map," IEEE Trans. Circuits Syst. I: Reg. Papers, vol. 54, no. 4, pp. 816-828, 2007.

[2] S.-L. Chen, T. Hwang, and W.-W. Lin, "Randomness enhancement using digitalized modified logistic map," IEEE Trans. Circuits Syst. II: Exp. Briefs, vol. 57, no. 12, pp. 996-1000, 2010.

[3] C.-Y. Li, T.-Y. Chang, and C.-C. Huang, "A nonlinear PRNG using digitized logistic map with self-reseeding method," Proc. IEEE Int. Symp. VLSI Design, Automation Test (VLSI-DAT), pp. 108-111, 2010.

[4] S.-L. Chen, T. Hwang, S.-M. Chang, and W.-W. Lin, "A fast digital chaotic generator for secure communication," Int. J. Bifurcation and Chaos, vol. 20, no. 12, pp. 1-19, 2010.

[5] M. A. Zidan, A. G. Radwan, and K. N. Salama, "Random number generation based on digital differential chaos," Proc. IEEE Int. Midwest Symp. Circuits Syst. (MWSCAS), pp. 1-4, 2011.

[6] C.-Y. Li, Y.-H. Chen, T.-Y. Chang, L.-Y. Deng, and K. To, "Period extension and randomness enhancement using high-throughput reseedingmixing PRNG," IEEE Trans. Very Large Scale Integr. (VLSI) Syst., vol. 20, no. 2, pp. 385-389, 2012.

[7] M. Delgado-Restituto and A. Rodríguez-Vázquez, "Integrated chaos generator," Proc. IEEE, vol. 90, no. 5, pp. 747-767, 2002

[8] T. Addabbo, M. Alioto, A. Fort, S. Rocchi, and V. Vignoli, "A feedback strategy to improve the entropy of a chaos-based random bit generator," IEEE Trans. Circuits Syst. I: Reg. Papers, vol. 53, no. 2, pp. 326 - 337, 2006.

[9] M. E. Yalçin, J. A. K. Suykens, and J. Vandewalle, "Experimental confirmation of 3- and 5-scroll attractors from a generalized Chua's circuit," IEEE Trans. Circuits Syst. I: Fundam. Theory Appl., vol. 47, no. 3, pp. 425-429, 2000.

[10] J. Lü and G. Chen, "Generating multiscroll chaotic attractors: Theories, methods and applications," Int. J. Bifurcation and Chaos, vol. 16, no. 4, pp. 775-858, 2006.

[11] K. N. Salama, S. Ozoguz, and A. S. Elwakil, "Generation of nscroll attractors using nonlinear transconductors," Proc. IEEE Int. Symp. Circuits Syst. (ISCAS), pp. 176-179, 2003.

[12] M. A. Zidan, A. G. Radwan, and K. N. Salama, "Controllable V-shape multi-scroll butterfly attractor: System and circuit implementation," Int. J. Bifurcation and Chaos, vol. 22, no. 6, 2012.

[13] J. Lü, S. Yu, and G. Chen, "Experimental verification of multidirectional multiscroll attractors," IEEE Trans. Circuits Syst. I: Reg. Papers, vol. 53, no. 1 , pp. $149-165,2006$.

[14] A. G. Radwan, A. M. Soliman, and A. S. Elwakil, "1-D digitallycontrolled multiscroll chaos generator," Int. J. Bifurcation and Chaos, vol. 17, no. 1, pp. 227-242, 2007.

[15] A. S. Mansingka, A. G. Radwan, M. A. Zidan, and K. N. Salama, "Analysis of bus width and delay on a fully digital signum nonlinearity chaotic oscillator," Proc. IEEE Int. Midwest Symp. Circuits Syst. (MWSCAS), pp. $1-4,2011$.

[16] A. S. Mansingka, A. G. Radwan, and K. N. Salama, "Design, implementation and analysis of fully digital 1-D controllable multiscroll chaos," Proc. Int. Conf. Microelectronics (ICM), pp. 1-5, 2011.

[17] M. A. Zidan, A. G. Radwan, and K. N. Salama, "The effect of numerical techniques on differential equation based chaotic generators," Proc. Int. Conf. Microelectronics (ICM), pp. 1-4, 2011.

[18] J. C. Sprott, "A new class of chaotic circuit," Physics Lett. A, vol. 266, pp. 19-23, 2000.

[19] S. Kodba, M. Perc, and M. Marhl, "Detecting chaos from a time series," European J. Physics, vol. 26, no. 1, pp. 205-215, 2005.

[20] A. Rukhin, J. Soto, M. Smid, E. Barker, S. Leigh, M. Levenson, M. Vangel, D. Banks, A. Heckert, J. Dray, S. Vo, and L. E. Bassham, "A statistical test suite for random and pseudorandom number generators for cryptographic applications," NIST Special Publication 800-22, 2010. 